\title{
Análise da prevalência de polifarmácia e do perfil farmacoterapêutico de idosos adscritos em uma unidade de saúde da família
}

\author{
Analysis of the prevalence of polypharmacy and the pharmacotherapeutic profile of elderly people \\ enrolled in a family health unit
}

Análisis de la prevalencia de polifarmacia y perfil farmacoterapéutico de personas mayores inscritas en una unidad de salud familiar

Recebido: 09/07/2021 | Revisado: 17/07/2021 | Aceito: 19/07/2021 | Publicado: 26/07/2021

\author{
Andressa Andrade Silva \\ ORCID: https://orcid.org/0000-0002-7535-153X \\ Universidade Estadual do Sudoeste da Bahia, Brasil \\ E-mail: andreessaandradee@gmail.com \\ Gisele da Silveira Lemos \\ ORCID: https://orcid.org/0000-0001-8987-0245 \\ Universidade Estadual do Sudoeste da Bahia, Brasil \\ E-mail: giselesilveiralemos@gmail.com \\ Tuany Santos Souza \\ ORCID: https://orcid.org/0000-0003-0165-4201 \\ Universidade Estadual do Sudoeste da Bahia, Brasil \\ E-mail: tuanysouza.s@uesb.edu.br
}

\begin{abstract}
Resumo
Objetivo: analisar a prevalência da polifarmácia e o perfil farmacoterapêutico de idosos adscritos em uma unidade de saúde da família. Métodos: Estudo farmacoepidemiológico transversal, quantitativo, descritivo e analítico, realizado com indivíduos com 60 anos ou mais no município de Lafaiete Coutinho - BA. A associação entre as variáveis foi testada usando a Regressão de Poisson, com cálculo robusto de razões de prevalência (RP) e intervalo de confiança de 95\% (IC95\%) e significância p<0,05. Resultados: Participaram 70 idosos; a prevalência da polifarmácia foi de 24,3\% e mostrou-se significativamente associada ao uso dos serviços de saúde pelo menos uma vez por ano ( $\mathrm{RP}=1,44$; $\mathrm{IC}=0,29-3,67 ; \mathrm{p}<0,001)$ ou por mais de duas vezes ao ano $(\mathrm{RP}=3,43 ; \mathrm{IC}=1,61-7,43 ; \mathrm{p}<0,01)$; aos idosos que não conhecem a indicação dos seus medicamentos $(\mathrm{RP}=7,40 ; \mathrm{IC}=1,37-39,89 ; \mathrm{p}=0,02)$, aos que relataram queixas sobre medicamentos $(\mathrm{RP}=4,09 ; \mathrm{IC}=0,78-21,42 ; \mathrm{p}=0,04)$ e aos que necessitam de algum recurso pra lembrar de tomar os seus medicamentos $(\mathrm{RP}=4,17 ; \mathrm{IC}=0,97-17,94 ; \mathrm{p}=0,05)$. $\mathrm{O}$ fator ajuda para tomar os medicamentos mostrou-se como fator de proteção $(\mathrm{RP}=0,19 ; \mathrm{IC}=0,03-1,00 ; \mathrm{p}=0,02)$. Conclusão: A prevalência da polifarmácia foi considerável e sugere a necessidade de uma maior sensibilização por parte dos profissionais de saúde, no que compete ao acompanhamento dos potenciais riscos e benefícios associados, afim de garantir o uso seguro dos medicamentos em regimes de polifarmácia nestes idosos.
\end{abstract}

Palavras-chave: Polifarmácia; Idoso; Perfil farmacoterapêutico; Medicamentos.

\begin{abstract}
Objective: to analyze the prevalence of polypharmacy and the pharmacotherapeutic profile of elderly people enrolled in a family health unit. Methods: Cross-sectional, quantitative, descriptive and analytical pharmacoepidemiological study, carried out with individuals aged 60 years or more in the city of Lafaiete Coutinho - BA. The association between variables was tested using Poisson Regression, with robust calculation of prevalence ratios (PR) and 95\% confidence interval $(95 \% \mathrm{CI})$ and significance $\mathrm{p}<0.05$. Results: 70 elderly people participated; the prevalence of polypharmacy was $24.3 \%$ and was significantly associated with the use of health services at least once a year $(\mathrm{PR}=1.44 ; \mathrm{CI}=0.29-3.67 ; \mathrm{p}<0.001)$ or for more than twice a year $(\mathrm{PR}=3.43 ; \mathrm{CI}=1.61-7.43 ; \mathrm{p}<0.01)$; to the elderly who do not know the indication of their medications ( $\mathrm{PR}=7.40 ; \mathrm{CI}=1.37-39.89 ; \mathrm{p}=0.02)$, to those who reported complaints about medications $(\mathrm{PR}=4.09 ; \mathrm{CI}=0 . .78-21.42 ; \mathrm{p}=0.04)$ and those who need some resource to remember to take their medications $(\mathrm{PR}=4.17 ; \mathrm{CI}=0.97-17.94 ; \mathrm{p}=0.05)$. The factor helping to take the medication was shown to be a protective factor $(\mathrm{PR}=0.19 ; \mathrm{CI}=0.03-1.00 ; \mathrm{p}=0.02)$. Conclusion: The prevalence of polypharmacy was considerable and suggests the need for greater awareness on the part of health professionals, regarding the monitoring of potential risks and associated benefits, in order to ensure the safe use of drugs in polypharmacy regimens in these elderly people.
\end{abstract}

Keywords: Polypharmacy; Elderly; Pharmacotherapeutic profile; Medicines. 


\begin{abstract}
Resumen
Objetivo: analizar la prevalencia de polifarmacia y el perfil farmacoterapéutico de los ancianos inscritos en una unidad de salud familiar. Métodos: Estudio farmacoepidemiológico transversal, cuantitativo, descriptivo y analítico, realizado con individuos de 60 años o más en la ciudad de Lafaiete Coutinho - BA. La asociación entre variables se probó mediante regresión de Poisson, con un cálculo robusto de las razones de prevalencia (RP) y el intervalo de confianza del 95\% (IC del 95\%) y significación p <0,05. Resultados: participaron 70 ancianos; la prevalencia de polifarmacia fue del $24,3 \%$ y se asoció significativamente con el uso de los servicios de salud al menos una vez al año $(\mathrm{RP}=1,44 ; \mathrm{IC}=0,29-3,67 ; \mathrm{p}<0,001)$ o más de dos veces al año $(\mathrm{RP}=3,43 ; \mathrm{IC}=1,61-7,43 ; \mathrm{p}<0,01)$; a los ancianos que desconocen la indicación de sus medicamentos ( $\mathrm{RP}=7,40 ; \mathrm{IC}=1,37-39,89 ; \mathrm{p}=0,02)$, a los que informaron quejas sobre medicamentos $(\mathrm{RP}=4,09 ; \mathrm{IC}=0.78-21,42 ; \mathrm{p}=0,04)$ y aquellos que necesitan algún recurso para acordarse de tomar sus medicamentos $(\mathrm{RP}=4,17 ; \mathrm{IC}=0,97-17,94 ; \mathrm{p}=0,05)$. Se demostró que el factor que ayuda a tomar la medicación es un factor protector $(\mathrm{PR}=0,19 ; \mathrm{IC}=0,03-1,00 ; \mathrm{p}=0,02)$. Conclusión: La prevalencia de la polifarmacia fue considerable y sugiere la necesidad de una mayor concienciación por parte de los profesionales de la salud, en cuanto al seguimiento de los posibles riesgos y beneficios asociados, con el fin de garantizar el uso seguro de los fármacos en los regímenes de polifarmacia en estas personas mayores.
\end{abstract}

Palabras clave: Polifarmacia; Anciano; Perfil farmacoterapéutico; Medicamentos.

\title{
1. Introdução
}

Tendo em vista o processo de envelhecimento da população com amplitude mundial, caracterizado pelo fenômeno da transição demográfica (WHO, 2002), no Brasil, estimativas feitas pelo Instituto Brasileiro de Geografia e Estatística (IBGE), indicam que indivíduos acima de 65 anos passarão da margem de 8,77\% do total da população em 2018 para aproximadamente 13,44\% em 2030 (IBGE, 2018). É relevante considerar que de forma adjacente observou-se também o processo de transição epidemiológica, onde houve um aumento das doenças crônico-degenerativas, em detrimento das doenças infecciosas e parasitárias. Neste sentido, as doenças crônicas não transmissíveis (DCNTs) apresentam altas prevalências em idosos e, no Brasil, chegam a representar a principal causa de mortalidade nesse grupo etário (Veras, 2011).

Além disso, as próprias alterações fisiológicas oriundas da idade, combinadas com inatividade física, tornam os idosos mais propensos a desenvolver esses tipos de enfermidades, causando uma perda progressiva da sua autonomia e independência (Fechini \& Trompieri, 2015). Entre as complicações de sistemas fisiológicos podem-se destacar as alterações cutâneas e musculoesqueléticas que causam dor e dificuldade de locomoção; alterações neurológicas, que levam gradualmente ao declínio de memória; comprometimento no sistema reprodutor, nos órgãos sensoriais, além de problemas auditivos e oftalmológicos (Ribeiro, Alves \& Meira, 2009). Diante deste quadro, a necessidade do consumo de medicamentos é cada vez maior em manifestações clínicas decorrentes do envelhecimento, principalmente para o tratamento de DCNTs. Assim, os indivíduos desta faixa etária, estão mais expostos a polifarmácia, sendo essa prática definida como o uso de cinco ou mais medicamentos, de acordo com a Organização Mundial de Saúde, (OMS) (WHO, 2019).

Esse fenômeno começou a tornar-se evidente como um problema de segurança relacionado ao uso de medicamentos (Lucchetti et al., 2010), o que torna esses usuários mais susceptíveis aos perigos que envolvem a farmacoterapia e contribui para um maior risco de reações adversas, interações medicamentosas e erros de medicação, sendo que os principais elementos responsáveis por esta prática constituem o estado funcional, as doenças crônicas e as inúmeras manifestações clínicas decorrentes do envelhecimento (Guimarães et al., 2012; Macedo, Rodrigues \& Correa, 2016; Rodrigues et al., 2021). Neste interim, um estudo realizado com idosos usuários do sistema público de saúde, evidenciou maior chance de comprometimento das funções renais, principalmente a alteração da taxa de filtração glomerular, associado a uma alta frequência de uso de polifarmácia e medicamentos potencialmente inapropriados para idosos, apontando para a necessidade de se investir em abordagens terapêuticas mais seguras (Morais et al., 2021).

Neste contexto, é importante elencar estratégias que visem o cuidado ao paciente e segurança no uso de medicamentos, como discutido por Ferreira e Soler (2020) em seu estudo, no sentido de contribuir diretamente na melhoria dos processos assistenciais. No que se refere especificamente aos idosos, destaca-se a necessidade de acompanhar a utilização de 
medicamentos nessa população de risco através da avaliação do perfil farmacoterapêutico (PF), sendo esta uma importante ferramenta que permite aos profissionais de saúde, em especial ao farmacêutico realizarem o acompanhamento do paciente e contribuir para o uso seguro e efetivo dos medicamentos (CFF, 2008).

Assim, tendo em vista que o amplo consumo de medicamentos na população idosa pode acarretar eventos adversos e com isso causar danos à sua saúde, tornam-se necessários mais estudos que investiguem quais as principais classes terapêuticas que estão sendo rotineiramente utilizadas por estes indivíduos, para que se possa planejar ações exequíveis na atenção básica, no que compete ao monitoramento desses idosos submetidos à esquemas terapêuticos com múltiplos medicamentos.

Com isto, tem-se a possibilidade de uma atuação profissional mais assistencialista com a finalidade de auxiliar os idosos à uma melhor adesão ao tratamento, menores riscos de reações adversas e problemas relacionados à farmacoterapia (PRFs) e, sobretudo, uma melhor qualidade de vida. Nesta perspectiva, este estudo tem como objetivo analisar a prevalência da polifarmácia e o perfil farmacoterapêutico de idosos adscritos em uma unidade de saúde da família.

\section{Metodologia}

Trata-se de um estudo farmacoepidemiológico, de delineamento transversal, de caráter quantitativo, descritivo e analítico. O campo do estudo foi o município de Lafaiete Coutinho, localizado na Mesorregião do Centro-Sul da Bahia, com um território de $353 \mathrm{~km}^{2}$ e localizado a $356 \mathrm{~km}$ da capital do Estado da Bahia. Segundo estimativas do IBGE (2018), tem uma população de 3.975 pessoas, e aproximadamente $14,94 \%$ dessas pessoas tem idade superior a 60 anos. O município possui apenas duas Unidades de Saúde da Família (USF) que atendem toda a população, entretanto o rastreamento dos idosos foi realizado em uma das USFs pelo fato de cobrir as microáreas de abrangência do centro da cidade.

Foram incluídos no estudo indivíduos de ambos os sexos, que apresentavam idade igual ou superior a 60 anos, residentes na zona urbana do município de Lafaiete Coutinho, não institucionalizados, com cognição preservada avaliada através do mini exame do estado mental (MEEM), proposto por Folstein, Folstein e Mchugh (1975); que utilizasse pelo menos um medicamento de uso contínuo no período da pesquisa e que aceitasse participar voluntariamente do estudo, mediante assinatura do termo de consentimento livre e esclarecido - TCLE.

Foram excluídos idosos que não possuíam condições de responder ao questionário ou que não tivessem acompanhante que os auxiliasse; idosos que apresentaram déficit cognitivo mediante avaliação do MEEM, com pontuação <13 pontos; idosos residentes na zona rural; aqueles que não foram encontrados em suas residências após três tentativas em dias e horários alternados. Assim, no total foram identificados 94 idosos cadastrados na USF de rastreio, totalizando 24 perdas, das quais 2 foram relacionadas a recusa, 1 por óbito, 1 por apresentar déficit cognitivo (MEEM $<13$ ), 2 por mudança de cidade e 18 por não terem sido encontrados em suas residências após três tentativas em dias e horários alternados. O diagrama do processo de inclusão dos idosos no estudo encontra-se na Figura 1. 
Figura 1 - Diagrama do processo de inclusão dos idosos no estudo. Lafaiete Coutinho-BA, Brasil, 2019.

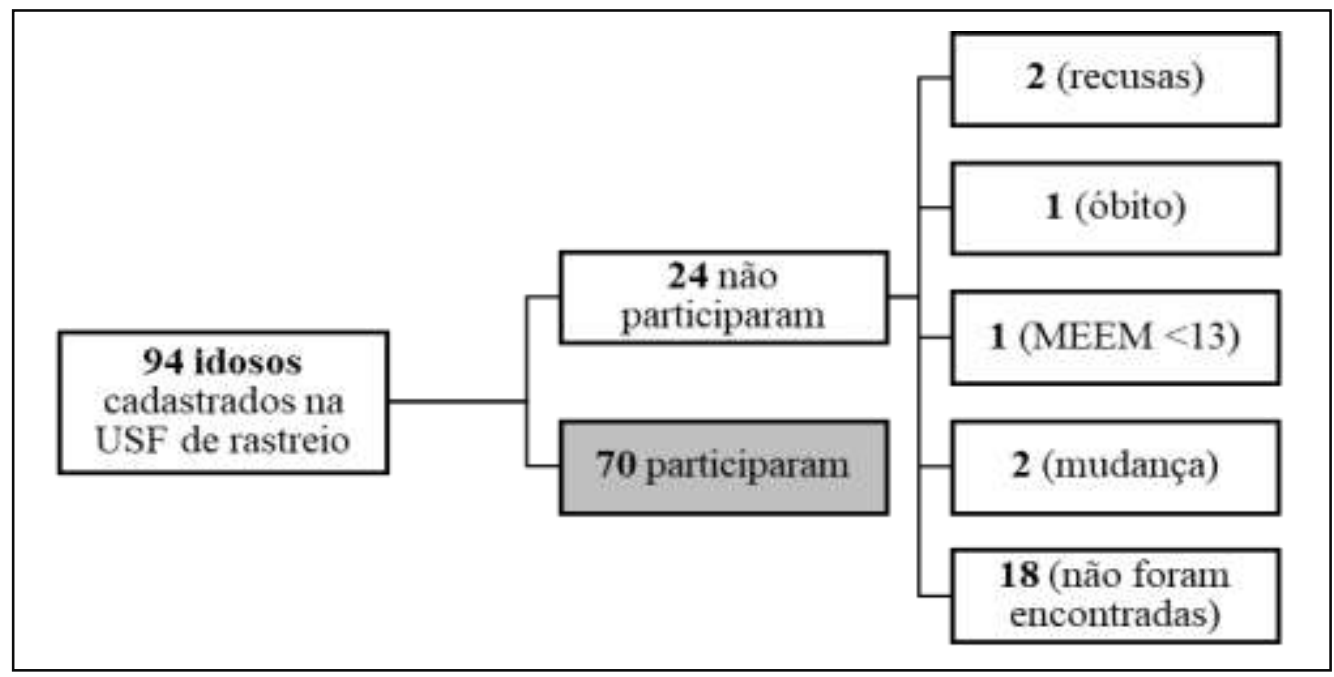

Fonte: Autores.

A coleta de dados foi realizada através de visitas domiciliares após triagem dos idosos na USF da zona urbana do município de Lafaiete Coutinho-BA através da aplicação de um questionário semiestruturado onde foram avaliadas as características sociodemográficas, condições de saúde, estilo de vida, bem como a utilização de medicamentos.

A variável dependente do estudo foi a polifarmácia definida através da observação da utilização de cinco ou mais medicamentos, de acordo com a OMS (WHO, 2019). As variáveis independentes foram as características sociodemográficas (sexo, idade, raça, renda familiar, situação conjugal, escolaridade); condições de saúde e estilo de vida (doenças autorreferidas, autopercepção de saúde, uso dos serviços de saúde, internações nos últimos 12 meses, consumo de bebidas alcoólicas, tabagismo, hábitos alimentares, prática de atividade física); bem como o perfil farmacoterapêutico (conhecimento sobre a indicação do medicamento, queixas relacionadas a medicamentos, uso de medicamentos por conta própria, uso de recursos para lembrar da utilização dos medicamentos, hábito de ler a bula, formas de aquisição do medicamento, dificuldade de acesso à medicamentos pelo SUS, dentre outros). Os medicamentos em uso e seus respectivos princípios ativos foram classificados de acordo com a Classificação Anatomical Therapeutic Chemical (ATC) da OMS, primeiro nível (WHO, 2000).

$\mathrm{Na}$ análise descritiva dos dados, a variável dependente foi expressa através do cálculo de prevalência, levando-se em consideração a população estudada ( $n^{\circ}$ total de idosos do estudo) e os expostos ( $n^{\circ}$ de idosos em uso de polifarmácia). As variáveis independentes foram expressas através frequência relativa e absoluta para as variáveis categóricas, e medidas de tendência central (média e desvio padrão) para as variáveis contínuas. A normalidade foi testada através do Teste de Kolmogorov-Sminorv.

Para testar a associação entre as variáveis independentes e o desfecho foi realizada a análise bivariada usando a Regressão de Poisson, com cálculo robusto de razões de prevalência (RP) e intervalo de confiança de 95\% (IC95\%), incluindo as variáveis no modelo de forma hierarquizada, afim de realizar ajustes intraníveis entre as características sociodemográficas (nível 1), condições de saúde e estilo de vida (nível 2) e perfil famacoterapêutico (nível 3). Os dados foram tabulados inicialmente em planilhas do software Microsoft Excel® e, para as análises estatísticas foi utilizado o software Statistical Package for the Social Sciences (SPSS), versão 21.0.

O presente estudo buscou atender aos preceitos e diretrizes da Resolução no 466 do Conselho Nacional de Saúde (CNS), de 12 de dezembro de 2012 (Brasil, 2012) e foi aprovado pelo Comitê de Ética da Universidade Estadual do Sudoeste da Bahia (CEP/UESB), sob CAAE 00846818.6.0000.0055 e número do parecer 3.050.079. 


\section{Resultados}

Participaram da pesquisa 70 idosos cadastrados em uma Unidade de Saúde da Família do município de Lafaiete Coutinho - BA entre dezembro de 2018 e janeiro de 2019, sendo a maioria do sexo feminino (71,4\%). A idade dos idosos variou de 60 a 93 anos, com média de idade de 72,6 anos (DP = 9,7), sendo faixa etária predominante a de idosos entre 60-69 anos (45,7\%). Quanto a escolaridade, houve um predomínio de idosos com menos de 8 anos de estudo (55,7\%) e que nunca estudaram $(24,29 \%)$.

Entre os idosos entrevistados, 62,9\% não eram negros, $80 \%$ recebiam até um salário mínimo, 82,9\% moravam acompanhados, 87,1\% eram aposentados ou pensionistas, 67,1\% alegaram ter a saúde boa e 91,4\% disseram que não tem dificuldade de acessar os serviços de saúde. As doenças mais frequentes foram Hipertensão Arterial Sistêmica (HAS) (92,9\%), Dislipidemia $(21,4 \%)$ e Diabetes Mellitus (17,1\%). As demais características sociodemográficas, comportamentais e de condições da saúde dos idosos estudados encontram-se descritas na Tabela 1.

Tabela 1 - Características sociodemográficas, comportamentais e de condições da saúde dos idosos. Lafaiete Coutinho-BA, Brasil, 2019.

\begin{tabular}{|c|c|c|c|}
\hline Variáveis & $\%$ resposta & $\mathbf{N}$ & $\%$ \\
\hline Sexo & 100 & & \\
\hline Masculino & & 20 & 28,6 \\
\hline Feminino & & 50 & 71,4 \\
\hline Faixa etária & 100 & & \\
\hline $60-69$ & & 32 & 45,7 \\
\hline 70-79 & & 19 & 27,1 \\
\hline$\geq 80$ & & 19 & 27,1 \\
\hline Com quem mora & 100 & & \\
\hline Acompanhante & & 58 & 82,9 \\
\hline Sozinho (a) & & 12 & 17,1 \\
\hline Situação Conjugal & 100 & & \\
\hline Com companheiro (a) & & 33 & 47,1 \\
\hline Sem companheiro (a) & & 37 & 52,9 \\
\hline Cor da pele & 100 & & \\
\hline Negro & & 26 & 37,1 \\
\hline Não Negro & & 44 & 62,9 \\
\hline Profissão & 100 & & \\
\hline Ativa & & 6 & 8,6 \\
\hline Aposentado/Pensionista & & 61 & 87,1 \\
\hline Nenhuma & & 3 & 4,3 \\
\hline Escolaridade (anos de estudo) & 100 & & \\
\hline$>8$ anos & & 14 & 20,0 \\
\hline$<8$ anos & & 39 & 55,7 \\
\hline Nunca estudou & & 17 & 24,3 \\
\hline Renda* & 100 & & \\
\hline > 1 salário mínimo & & 15 & 21,4 \\
\hline$\leq 1$ salário mínimo & & 52 & 74,3 \\
\hline Nenhuma & & 3 & 4,3 \\
\hline Doenças auto referidas & 100 & & \\
\hline Nenhuma & & 0 & 0,0 \\
\hline Apenas 1 & & 27 & 38,6 \\
\hline 2 ou mais & & 43 & 61,4 \\
\hline Auto percepção de saúde & 100 & & \\
\hline Excelente & & 0 & 0,0 \\
\hline Boa & & 47 & 67,1 \\
\hline Regular/Ruim & & 23 & 32,9 \\
\hline Uso de serviços de saúde & 100 & & \\
\hline Nenhuma & & 20 & 28,6 \\
\hline
\end{tabular}


Research, Society and Development, v. 10, n. 9, e29210918069, 2021

(CC BY 4.0) | ISSN 2525-3409 | DOI: http://dx.doi.org/10.33448/rsd-v10i9.18069

\begin{tabular}{|c|c|c|c|}
\hline 1 vez/ano & & 9 & 12,9 \\
\hline 2 ou mais & & 41 & 58,6 \\
\hline Dificuldade de acessar os serviços de saúde & 100 & & \\
\hline Não & & 64 & 91,4 \\
\hline Sim & & 6 & 8,6 \\
\hline Internações nos últimos 12 meses & 100 & & \\
\hline Nenhuma & & 59 & 84,3 \\
\hline 1 ou mais & & 11 & 15,7 \\
\hline Plano de saúde & 100 & & \\
\hline Sim & & 8 & 11,4 \\
\hline Não & & 62 & 88,6 \\
\hline Consumo de álcool & 100 & & \\
\hline Não & & 61 & 87,1 \\
\hline Sim & & 9 & 12,9 \\
\hline Tabagismo & 100 & & \\
\hline Não & & 68 & 97,1 \\
\hline Sim & & 2 & 2,9 \\
\hline Alimentação por dia & 100 & & \\
\hline 3 ou mais refeições regulares & & 66 & 94,3 \\
\hline Apenas 2 refeições regulares & & 4 & 5,7 \\
\hline Atividades físicas & 100 & & \\
\hline $3 \mathbf{x} / \mathbf{s e m}$ & & 6 & 8,6 \\
\hline $1-3 x /$ sem & & 10 & 14,3 \\
\hline Não pratica & & 54 & $\mathbf{7 7 , 1}$ \\
\hline
\end{tabular}

Fonte: Autores.

A descrição do perfil farmacoterapêutico dos idosos encontra-se na Tabela 2, onde observa-se que a prevalência da polifarmácia nesta população foi de $24,3 \%$, envolvendo um total de 241 medicamentos. Foi possível verificar que o número máximo de medicamentos utilizado por um idoso foi de 10 , com média de 3,5 medicamentos por pessoa $(\mathrm{DP}=1,8)$, sendo que $67,1 \%$ usavam pelo menos um medicamento por conta própria.

Dos idosos entrevistados, 95,7\% informaram ter conhecimento sobre a indicação dos medicamentos que faziam uso e a maioria não relataram queixas relacionadas aos mesmos $(95,7 \%)$, no entanto, dos poucos idosos que relataram queixas, as principais foram: sonolência, insônia e palpitações.

No que se refere à necessidade de ajuda para tomar os medicamentos, cerca de $87,1 \%$ relataram que não necessitam e afirmaram lembrar de usá-los na hora certa, demonstrando serem bem instruídos sobre o seu tratamento. A principal forma de aquisição dos medicamentos utilizados são as Farmácias Privadas (52,9\%). Dentre os entrevistados, 58,6\% afirmaram ter dificuldade de acesso aos seus medicamentos pelo SUS, no entanto 98,6\% não deixam de utilizá-los em virtude da falta de acesso gratuito. 
Tabela 2 - Prevalência da polifarmácia e perfil farmacoterapêutico de idosos. Lafaiete Coutinho-BA, Brasil, 2019.

\begin{tabular}{|c|c|c|c|}
\hline Variáveis & \% resposta & $\mathbf{N}$ & $\%$ \\
\hline Polifarmácia & 100 & & \\
\hline Não & & 53 & 75,7 \\
\hline Sim & & 17 & 24,3 \\
\hline Conhecimento da indicação do medicamento & 100 & & \\
\hline Não & & 3 & 4,3 \\
\hline Sim & & 67 & 95,7 \\
\hline Queixa relacionada a algum medicamento & 100 & & \\
\hline Não & & 67 & 95,7 \\
\hline Sim & & 3 & 4,3 \\
\hline Necessidade de ajuda para tomar os medicamentos & 100 & & \\
\hline Não & & 61 & 87,1 \\
\hline Sim & & 9 & 12,9 \\
\hline Lembra-se de tomar os medicamentos na hora certa & 100 & & \\
\hline Às vezes/não & & 9 & 12,9 \\
\hline Sim & & 61 & 87,1 \\
\hline Utiliza recurso para lembrar de tomar os medicamentos & 100 & & \\
\hline Não & & 58 & 82,9 \\
\hline Sim & & 12 & 17,1 \\
\hline Sabe onde estão guardados os medicamentos & 100 & & \\
\hline Não & & 5 & 7,1 \\
\hline Sim & & 65 & 92,9 \\
\hline Utilização de algum medicamento por conta própria & 100 & & \\
\hline Não & & 23 & 32,9 \\
\hline Sim & & 47 & 67,1 \\
\hline Cessação do uso de algum medicamento ao sentir piora & 100 & & \\
\hline Não & & 56 & 80,0 \\
\hline Sim & & 14 & 20,0 \\
\hline Mudança de horário de algum medicamento por conta própria & 100 & & \\
\hline Não & & 62 & 88,6 \\
\hline Sim & & 8 & 11,4 \\
\hline Mudança da dose de algum medicamento por conta própria & 100 & & \\
\hline Não & & 69 & 98,6 \\
\hline Sim & & 1 & 1,4 \\
\hline Percepção de que não precisa de algum medicamento que faz uso & 100 & & \\
\hline Não & & 66 & 94,3 \\
\hline Sim & & 4 & 5,7 \\
\hline Percepção de que precisa de algum medicamento que não faz uso & 100 & & \\
\hline Não & & 60 & 85,7 \\
\hline Sim & & 10 & 14,3 \\
\hline Hábito de ler bulas de medicamentos & 100 & & \\
\hline Não & & 58 & 82,9 \\
\hline Sim & & 12 & 17,1 \\
\hline Confunde medicamentos que usa & 100 & & \\
\hline Não & & 67 & 95,7 \\
\hline Sim & & 3 & 4,3 \\
\hline Forma de aquisição de medicamentos & 100 & & \\
\hline Unidades Básicas de Saúde & & 28 & 40,0 \\
\hline Farmácias Privadas & & 37 & 52,9 \\
\hline Aqui tem Farmácia Popular & & 5 & 7,1 \\
\hline Dificuldade de acesso a medicamento pelo SUS & 100 & & \\
\hline Não & & 29 & 41,4 \\
\hline Sim & & 41 & 58,6 \\
\hline Deixa de usar o medicamento quando está em falta no SUS & 100 & & \\
\hline Não & & 69 & 98,6 \\
\hline Sim & & 1 & 1,4 \\
\hline
\end{tabular}

Fonte: Autores. 
Na Figura 2 encontram-se distribuídos os grupos farmacológicos utilizados pelos idosos deste estudo, classificados segundo o código ATC (nível 1). Verificou-se que as classes terapêuticas de maior prevalência foram os medicamentos para o Sistema Cardiovascular (62,2\%), seguido de medicamentos para o Trato Gastrointestinal e Metabolismo (13,3\%).

Figura 2 - Descrição dos medicamentos utilizados pelos idosos, segundo a classificação ATC, nível 1. Lafaiete Coutinho, Bahia, Brasil, 2019.

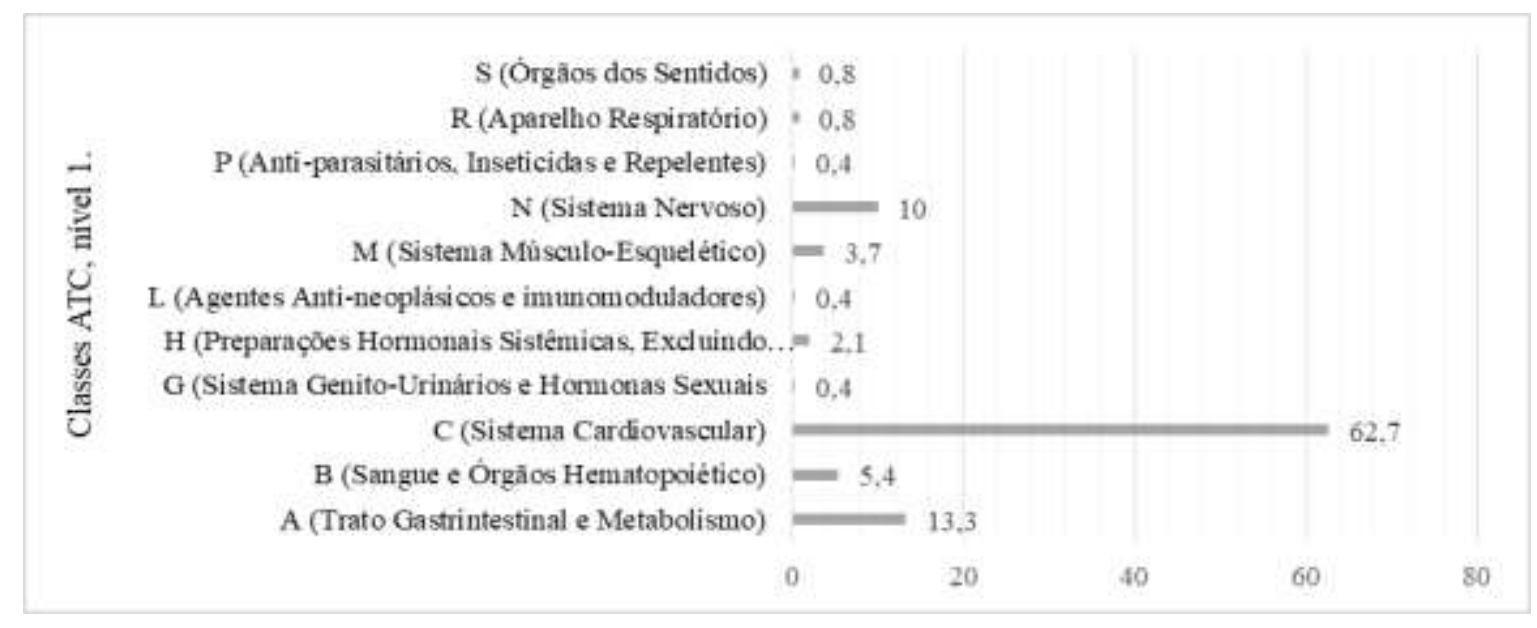

ATC: Anatomical Therapeutic Chemical. Fonte: Autores.

Na Tabela 3 é evidenciada a associação da polifarmácia com as variáveis independentes do estudo. Destaca-se que que a prevalência da polifarmácia nos idosos participantes deste estudo foi de $24,3 \%$ e mostrou-se significativamente associada ao uso dos serviços de saúde pelo menos uma vez por ano ( $\mathrm{RP}=1,44 ; \mathrm{IC}=0,29-3,67 ; \mathrm{p}<0,001)$ ou por mais de duas vezes ao ano ( $\mathrm{RP}=3,43 ; \mathrm{IC}=1,61-7,43 ; \mathrm{p}<0,01)$; aos idosos que não conhecem a indicação de seus tratamentos $(\mathrm{RP}=7,40 ; \mathrm{IC}=1,37-39,89$; $\mathrm{p}=0,02)$; aos que possuem alguma queixa relacionada aos medicamentos $(\mathrm{RP}=4,09 ; \mathrm{IC}=0,78-21,42 ; \mathrm{p}=0,04)$ e aos que necessitam de algum recurso pra lembrar de tomar os seus medicamentos ( $\mathrm{RP}=4,17 ; \mathrm{IC}=0,97-17,94 ; \mathrm{p}=0,05)$. A variável ajuda para tomar os medicamentos mostrou-se como fator de proteção ( $\mathrm{RP}=0,19 ; \mathrm{IC}=0,03-1,00 ; \mathrm{p}=0,02)$.

Tabela 3 - Prevalência de polifarmácia em idosos e sua associação com as variáveis independentes do estudo. Lafaiete Coutinho-BA, Brasil, 2019.

\begin{tabular}{|c|c|c|c|c|c|}
\hline Nível & Variáveis & Prevalência (\%) & RP* & IC $(95 \%)$ & p-valor \\
\hline \multirow{16}{*}{ 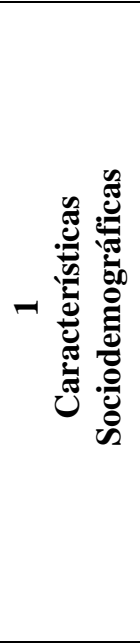 } & Sexo & & & & \\
\hline & Masculino & 20 & 1 & & \\
\hline & Feminino & 26 & 1,59 & $0,60-4,24$ & 0,350 \\
\hline & Faixa etária & & & & \\
\hline & $60-69$ & 25 & 1 & & \\
\hline & $70-79$ & 15,8 & 0,78 & $0,23-2,69$ & 0,692 \\
\hline & $\geq 80$ & 31,6 & 1,90 & $0,68-5,34$ & 0,222 \\
\hline & Arranjo Familiar & & & & \\
\hline & Com companheiro (a) & 30,3 & 1 & & \\
\hline & Sem companheiro (a) & 18,9 & 0,54 & $0,24-1,22$ & 0,140 \\
\hline & Cor da pele & & & & \\
\hline & Negro & 26,9 & 1 & & \\
\hline & Não negro & 22,7 & 0,67 & $0,27-1,67$ & 0,395 \\
\hline & Profissão & & & & \\
\hline & Ativa & 16,7 & 1 & & \\
\hline & Aposentado/pensionista & 25 & 1,87 & $0,29-12,29$ & 0,507 \\
\hline
\end{tabular}




\begin{tabular}{|c|c|c|c|c|c|}
\hline & \multicolumn{5}{|l|}{ Escolaridade } \\
\hline & $>8$ anos & 35,7 & 1 & & \\
\hline & $<8$ anos & 23,1 & 0,48 & $0,15-1,56$ & 0,221 \\
\hline & Nunca estudou & 17,6 & 0,29 & $0,04-2,04$ & 0,218 \\
\hline & \multicolumn{5}{|l|}{ Renda* } \\
\hline & > 1 salário mínimo & 26,7 & 1 & & \\
\hline & < 1 salário mínimo & 23,6 & 1,16 & $0,28-4,73$ & 0,840 \\
\hline \multirow{28}{*}{ 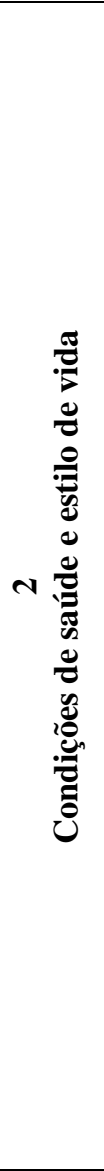 } & \multicolumn{5}{|l|}{ Doenças auto referidas } \\
\hline & Apenas 1 & 14,8 & 1 & & \\
\hline & 2 ou mais & 30,2 & 1,27 & $0,38-4,23$ & 0,699 \\
\hline & \multicolumn{5}{|l|}{ Auto percepção de saúde } \\
\hline & Excelente/Boa & 19,1 & 1 & & \\
\hline & Regular/Ruim & 34,8 & 1,06 & $0,45-2,53$ & 0,886 \\
\hline & \multicolumn{5}{|l|}{ Uso serviços de saúde } \\
\hline & Nenhuma & 0,0 & 1 & & \\
\hline & 1 vez/ano & 66,7 & 1,44 & $0,29-3,67$ & $<0,001$ \\
\hline & $\geq 2$ vezes/ano & 26,8 & 3,43 & $1,61-7,43$ & $<0,001$ \\
\hline & \multicolumn{5}{|l|}{ Internações nos últimos 12 meses } \\
\hline & Nenhuma & 20,3 & 1 & $0,68-2,96$ & 0,523 \\
\hline & 1 ou mais & 45,5 & 1,26 & $0,51-3,09$ & 0,617 \\
\hline & \multicolumn{5}{|l|}{ Plano de saúde } \\
\hline & Sim & 37,5 & 1 & & \\
\hline & Não & 22,6 & 0,73 & $0,25-2,14$ & 0,565 \\
\hline & \multicolumn{5}{|l|}{ Consumo de álcool } \\
\hline & Não & 26,2 & 1 & & \\
\hline & Sim & 11,1 & 0,60 & $0,13-2,75$ & 0,507 \\
\hline & \multicolumn{5}{|l|}{ Consumo de tabaco } \\
\hline & Não & 25 & 1 & & \\
\hline & Sim & 0 & 1,83 & $0,76-3,11$ & 0,304 \\
\hline & \multicolumn{5}{|l|}{ Alimentação por dia } \\
\hline & 3 ou mais refeições regulares & 57,8 & 1 & & \\
\hline & $\leq 2$ refeições regulares & 12,4 & 1,99 & $0,70-5,67$ & 0,196 \\
\hline & \multicolumn{5}{|l|}{ Atividades físicas } \\
\hline & Pratica & 31,3 & 1 & & \\
\hline & \multirow{2}{*}{\multicolumn{5}{|c|}{$\begin{array}{l}\text { Não pratica } \\
\text { Conhece a indicação do medicamento }\end{array}$}} \\
\hline \multirow{21}{*}{ の } & & & & & \\
\hline & Sim & 20,9 & 1 & & \\
\hline & Não & 100 & 7,40 & $1,37-39,89$ & 0,02 \\
\hline & \multicolumn{5}{|l|}{ Queixa relacionada a algum medicamento } \\
\hline & Não & 20,9 & 1 & & \\
\hline & Sim & 100 & 4,09 & $0,78-21,42$ & 0,04 \\
\hline & \multicolumn{5}{|l|}{$\begin{array}{l}\text { Necessita de ajuda para tomar para tomar } \\
\text { os medicamentos }\end{array}$} \\
\hline & Não & 24,6 & 1 & & \\
\hline & Sim & 22,2 & 0,19 & $0,03-1,00$ & 0,00 \\
\hline & \multicolumn{5}{|l|}{$\begin{array}{l}\text { Utiliza recurso para lembrar de tomar os } \\
\text { medicamentos }\end{array}$} \\
\hline & Não & 24,1 & 1 & & \\
\hline & Sim & 25 & 4,17 & $0,97-17,94$ & 0,05 \\
\hline & \multicolumn{5}{|l|}{$\begin{array}{l}\text { Utilização de algum medicamento por } \\
\text { conta própria }\end{array}$} \\
\hline & Não & 21,7 & 1 & & \\
\hline & Sim & 25,5 & 1,53 & $0,18-2,66$ & 0,273 \\
\hline & $\begin{array}{l}\text { Percepção de que precisa de algum } \\
\text { medicamento que não faz uso }\end{array}$ & & & & \\
\hline & Não & 18,3 & 1 & & \\
\hline & Sim & 60 & 1,48 & $0,29-7,39$ & 0,636 \\
\hline & Confunde medicamentos que usa & & & & \\
\hline & Não & 22,4 & 1 & & \\
\hline & Sim & 66,7 & 1,77 & $0,19-3,11$ & 0,715 \\
\hline
\end{tabular}




$\begin{array}{lcccc}\text { Forma de aquisição de medicamentos } & & & & \\ \quad \text { Unidades Básicas de Saúde } & 14,3 & 1 & & \\ \quad \text { Farmácias Privadas } & 35,1 & 1,52 & 0,32-4,21 & 0,345 \\ \quad \text { Farmácia Popular } & 12,2 & 1,09 & 0,27-2,67 & 0,409 \\ \text { Dificuldade de acesso a medicamento pelo } & & & & \\ \text { SUS } & 20,7 & 1 & & \\ \quad \text { Não } & 26,8 & 1,45 & 0,33-6,47 & 0,625 \\ \quad \text { Sim } & & & & \\ \text { Deixa de usar o medicamento quando está } & & & & \\ \text { em falta no SUS } & 24,6 & 1 & & \\ \quad \text { Não } & 9,3 & 1,09 & 0,42-3,89 & 0,213 \\ \quad \text { Sim } & \end{array}$

*Razão de prevalência. SUS: Sistema Único de Saúde. Fonte: Autores.

Observou-se ainda que 100\% dos idosos que relataram não ter conhecimento sobre a indicação do medicamento e que tem queixas relacionadas aos medicamentos fazem o uso da polifarmácia. Além disso, outras variáveis que possuem destaque, embora não tenham apresentado associação estaticamente significativa, são: a maior prevalência da polifarmácia em indivíduos que fazem uso de medicamentos por conta própria (25\%), assim como em indivíduos que confundem seus medicamentos $(66,7 \%)$.

A polifarmácia mostrou-se mais prevalente também nos idosos que relataram a principal forma de aquisição dos seus medicamentos em Farmácias Privadas $(35,1 \%)$, podendo isto estar relacionado com a dificuldade de obtenção de medicamentos pelo Sistema Único de Saúde (SUS) (26,8\%) e por esses indivíduos não deixarem de comprar o medicamento quando estão em falta na Unidade Básica de Saúde (24,6\%).

\section{Discussão}

A presente pesquisa realizada com idosos adscritos em uma Unidade de Saúde da Família do município de Lafaiete Coutinho-BA identificou uma prevalência de polifarmácia de 24,3\% nos idosos estudados. O elevado consumo de medicamentos pode ser justificado pela alta prevalência de DCNTs neste estrato da população (Sales, A., Sales, M. \& Casotti, 2010), corroborando com o autorrelato de agravos desta natureza, pelos idosos deste estudo, como HAS, dislipidemias e diabetes.

Ao comparar as prevalências da polifarmácia entre outros estudos realizados com idosos, utilizando os mesmos critérios para mensurá-la, foram identificados dados semelhantes, como por exemplo, em outro município de pequeno porte no interior da Bahia, onde a prevalência da polifarmácia foi de 29\% (Sales, Sales, \& Casotti, 2010). Prevalências superiores foram obtidas no município de Aracaju-SE [63,2\%] (Guimarães et al., 2012) e em Ribeirão Preto-SP [79\%] (Mercedes et al., 2013). Prevalência inferior foi obtida no município de Fortaleza-CE [13,6\%] (Coelho, Marcopito \& Castelo, 2004).

Tais divergências na prevalência da polifarmácia podem ser explicadas pelo fato de que o número de medicamentos utilizados pode ser influenciado pelas particularidades de cada região, no que diz respeito à fatores sociais, econômicos e tamanho do município, se pequeno ou grande porte. Além disso, outros fatores podem influenciar essas diferenças, dado que o estado de saúde e/ou condição do serviço de atenção à saúde dos municípios também são relevantes. Ademais, os recortes temporais distintos em que foram feitas as pesquisas, podem evidenciar variações nos medicamentos utilizados habitualmente pelos idosos na última semana, nos últimos meses ou ano.

A prevalência da polifarmácia neste estudo teve uma maior proporção entre mulheres, corroborando com outros estudos brasileiros, como o realizado em Belo Horizonte-MG (Loyola, Uchoa \& Lima-Costa, 2006) onde o uso de polifarmácia entre mulheres foi de 70,6\% e em Aiquara-BA (Sales, Sales, \& Casotti, 2010), onde a prática da polifarmácia em mulheres foi de $81,2 \%$. A maior utilização de medicamentos entre idosas pode estar relacionada com o fato de que utilizam com mais 
frequência os serviços de saúde, têm maior preocupação com sintomas físicos e são mais expostas a problemas de saúde não fatais, tendo assim, uma maior sobrevida (Carvalho et al., 2012).

Os medicamentos que agem sobre o aparelho cardiovascular foram os mais prevalentes entre os idosos de Lafaiete Coutinho-BA, em consonância com outros estudos em que dentre as DCNT que estão em maior número entre os idosos, grande parte conduz à necessidade da utilização de medicamentos que ajam no sistema cardiovascular (Sales, A., Sales, M. \& Casotti, 2010; Carvalho et al., 2012). Dentro dessa classe, os medicamentos mais utilizados foram Losartana (54,3\%) e Hidroclorotiazida $(47,1 \%)$, destinados para o tratamento da HAS, um dos mais importantes fatores de risco para doença renal crônica e acidentes cerebrovasculares (Martins et al., 2007; Dutra et al., 2016). Segundo a VII Diretriz Brasileira de Hipertensão, esta doença atinge mais de $60 \%$ da população idosa, e contribui direta ou indiretamente para 50\% das mortes por doença cardiovascular (SBC, 2016). Dado semelhante também foi descrito no estudo de Leão et al. (2020) e no estudo de Almeida et al. (2018), ambos realizados com pacientes hipertensos no contexto da atenção primária, em um município do interior da Bahia.

Um fato que chama atenção nesta população é a baixa escolaridade, onde a maioria dos idosos entrevistados têm menos de 8 anos de estudo, isto é, cursaram de forma completa ou incompleta apenas o ensino fundamental, enquanto outros nunca estudaram. Isso pode sugerir alguma relação com o baixo índice observado de consulta às bulas, acarretando em menor possibilidade aos idosos de acesso às informações pertinentes a seus tratamentos, levando-se em consideração ainda, a dificuldade de compreensão da linguagem técnica e rebuscada do conteúdo das bulas, onde muitos optam por não ler (Neto et al., 2012).

Destaca-se, ainda, que a maioria dos idosos $(67,1 \%)$ relatou fazer uso de medicamento por conta própria, devido ao fácil acesso para comprá-los, assim como também a prevalência da polifarmácia foi maior nestes indivíduos (25,5\%), quando comparados aos que não se automedicam. Particularmente em indivíduos nesta faixa etária as desvantagens da automedicação devem ser consideradas, entre elas pode se destacar diagnóstico e terapêutica inadequados, riscos de interações com medicamentos prescritos, reações adversas, intoxicação, resistência bacteriana, além de fortalecer a presença da polifarmácia (Oliveira et al., 2012).

Ademais, destaca-se neste estudo a associação entre a polifarmácia e a ocorrência de queixas relacionadas aos medicamentos $(\mathrm{RP}=4,09 ; \mathrm{IC}=0,78-21,42)$, denotando que os idosos que possuem alguma queixa relacionada aos medicamentos possuem probabilidade quatro vezes maior de estar em uso de polifarmácia do que os que não possuem nenhuma queixa. Neste estudo, as queixas mais descritas pelos idosos foram sonolência, já outros relataram insônia e/ou dificuldade para dormir e palpitações quando utilizam os medicamentos que necessitam. O estudo de Kadam (2011) evidenciou correlação entre o número de queixas, problemas de saúde e número de medicamentos utilizados, interferindo assim, nas condições de saúde dos indivíduos.

Além disso, neste estudo identificamos que o fato de os idosos não conhecerem a indicação dos medicamentos que usam, aumenta em sete vezes a probabilidade da ocorrência da polifarmácia ( $\mathrm{RP}=7,40 ; \mathrm{IC}=1,38-39,89$ ), assim como utilizar algum recurso para lembrar aumenta essa probabilidade em até quatro vezes $(\mathrm{RP}=4,17$; $\mathrm{IC}=0,97-17,94)$. Verifica-se com este achado a necessidade de atualização e acompanhamento dos idosos quanto a orientação sobre os seus medicamentos, principalmente pelos profissionais de saúde, que muitas vezes prescrevem e dispensam receitas sem prestar os devidos esclarecimentos ou orientações a este público, de forma especial (Araújo, 2011).

Assim, sugere-se que além da própria tendência dos idosos ao déficit cognitivo (Fechine \& Trompieri, 2015), a falta de orientação torna mais difícil o entendimento e conhecimento sobre a finalidade dos medicamentos que fazem uso, e isso consequentemente pode vir a ser uma agravante para a efetividade de seus tratamentos. Não obstante, o fato de não conhecerem os seus medicamentos pode expor esses idosos a PRFs, como tomar medicamentos que não necessitem ou ainda 
deixar de tomar os que necessitam, além do risco de ingerir doses excessivas e assim ficarem expostos a complicações mais graves associadas à polifarmácia, como interações medicamentosas, eventos adversos, dentre outros (Araújo, 2011; Marin et al., 2008).

Tais problemas, quando associados à baixa renda e baixa escolaridade, geralmente incompatíveis com as necessidades básicas dos idosos, atrelado às alterações cognitivas, funcionais e biopsicossociais da terceira idade (WHO, 2006) demonstram a complexidade acerca do uso de medicamentos entre os idosos e o quanto isso se torna um desafio para a assistência farmacêutica na atenção primária, pois mesmo que outras formas de cuidado sejam incorporadas pelas equipes de saúde, o medicamento ainda é uma das principais estratégias terapêuticas de enfrentamento das doenças crônicas que acometem a maioria dos indivíduos no processo de envelhecimento (Marin et al, 2008).

Por outro lado, observa-se que a variável ajuda para tomar os medicamentos mostrou-se como um fator de proteção para os idosos deste estudo ( $\mathrm{RP}=0,19 ; \mathrm{IC}=0,03-1,00)$, isso demonstra que os idosos que têm ajuda para administrar os seus medicamentos tem uma menor probabilidade de fazer o uso da polifarmácia, denotando a importância de um familiar ou acompanhante cuidador assumir a corresponsabilidade dos cuidados do idoso no que diz respeito ao uso correto de seus medicamentos. Segundo Barros, Silva e Leite (2015), uma das principais dificuldades para o uso seguro de medicamentos no que concerne a administração dos mesmos pelos idosos é a necessidade de negociação da rotina dos familiares e os horários de utilização desses medicamentos.

Por isso, julga-se importante uma parceria entre a família e os profissionais de saúde, para relatar as manifestações clínicas que o idoso possa apresentar. Ademais, essa ideia reforça a emergente necessidade de priorização de estratégias para promover a segurança no uso de medicamentos, elencando o farmacêutico como um ator-chave nesse processo, a fim de promover a segurança e qualidade de vida do paciente e a racionalização dos custos com tecnologias em saúde (Ferreira \& Soler, 2020).

Uma das limitações deste estudo é o fato de o delineamento transversal impossibilitar inferências sobre a temporalidade das associações encontradas. Cita-se ainda que a obtenção de dados por autorrelato, embora ainda seja a ferramenta mais utilizada no inquérito domiciliar (Carvalho et al., 2012), pode estar sujeita a viés de memória, considerando a subestimação de prevalências de algumas variáveis. No entanto, destaca-se que este estudo reforça a necessidade de acompanhamento da utilização de medicamentos por idosos, por parte dos profissionais da equipe multiprofissional de saúde na perspectiva da atenção primária, principalmente ações relacionadas a atuação do profissional farmacêutico no gerenciamento da qualidade e segurança da farmacoterapia do paciente idoso.

\section{Conclusão}

Conclui-se que a prevalência da polifarmácia encontrada nos idosos participantes deste estudo foi considerável e apresentou associação significativa com uso dos serviços de saúde pelo menos uma ou por mais de duas vezes ao ano; com o não conhecimento sobre a indicação dos seus tratamentos; com o relato de queixas relacionadas aos medicamentos e com a necessidade de utilização de recursos que auxiliem os idosos a lembrar de tomar os seus medicamentos. $\mathrm{O}$ fator ajuda para tomar os medicamentos mostrou-se como fator de proteção.

Considerando tais associações, sugere-se a necessidade de uma maior sensibilização por parte dos profissionais de saúde, no que compete ao acompanhamento dos potenciais riscos e benefícios associados, a fim de garantir o uso seguro dos medicamentos em regimes de polifarmácia nestes idosos. Estes achados reforçam também a necessidade de aprimorar as estratégias de cuidados farmacoterapêuticos nesta faixa etária, aliados à atenção domiciliar por parte dos familiares cuidadores, com foco na adoção adjacente de medidas preventivas e abordagens não farmacológicas. 


\section{Referências}

Almeida, P. H. R. F., Leão, I. N., Oliveira, B. G., Fernandes, B. D., Álvares, J., Silva, W. C., David, M. M., Lemos, G. S. \& Mascarenhas, C. H. M. (2018). Regime terapêutico e qualidade de vida de pacientes hipertensos. Rev. Aten. Saúde, 16(58), 17-28.

Araújo, C. L. (2011). Conhecimento de idosos sobre o uso de medicamentos e interação medicamentosa. RBCEH, Passo Fundo, 8(2), $188-195$.

Barros, D. S. L., Silva, D. L. M. \& Leite, S. N. (2015). Conduta do tratamento medicamentoso por cuidadores de idosos. Interface-Comunicação, Saúde, Educação, 19, 527-536.

Brasil. Ministério da Saúde. Conselho Nacional de Saúde. (2012). Resolução nº 466, de 12 de dezembro de 2012 . Diretrizes e normas regulamentadoras de pesquisas envolvendo seres humanos.

Carvalho, M. F. C., Romano-Lieber, N. S., Bergsten-Mendes, G., Secoli, S. R., Ribeiro, E., Lebrão, M. L. \& Duarte, Y. A. D. O. (2012). Polifarmácia entre idosos do município de São Paulo-Estudo SABE. Revista Brasileira de Epidemiologia, 15, 817-827.

Coelho Filho, J. M., Marcopito, L. F. \& Castelo, A. (2004). Perfil de utilização de medicamentos por idosos em área urbana do Nordeste do Brasil. Revista de Saúde Pública, 38, 557-564.

Conselho Federal de Farmácia (BR). (2008). Resolução no 499 de 10 de dezembro de 2008. Dispõe sobre a prestação de serviços farmacêuticos, em farmácias e drogarias, e dá outras providências. Brasília, Distrito Federal.

Dutra, D. D., Duarte, M. C. S., de Albuquerque, K. F., de Lima, A. S., de Souza Santos, J. \& Souto, H. C. (2016). Doenças cardiovasculares e fatores associados em adultos e idosos cadastrados em uma unidade básica de saúde Cardiovascular. Revista de Pesquisa: Cuidado é Fundamental Online, 8(2), 45014509.

Fechine, B. R. A. \& Trompieri, N. (2015). O processo de envelhecimento: as principais alterações que acontecem com o idoso com o passar dos anos. InterSciencePlace, 1(20).

Ferreira, AS., \& Soler, O. (2020). Fortalecendo as estratégias de segurança do paciente: uma revisão integrativa sobre os processos de segurança de medicamentos. Research, Society and Development, 9 (12), e129129564.

Folstein, M. F., Folstein, S. E. \& Mchugh, P. R. (1975). "Mini-mental state" A practical method for grading the cognitive state of patients for the clinician. Journal of Psychiatric Research, Standford, 12(3), 189-198.

Guimarães, V. G., Brito, G. D. C., Barbosa, L. D. M., Aguiar, P. M., Balisa-Rocha, B. J. \& Lyra Junior, D.P. (2012). Perfil Farmacoterapêutico de um Grupo de Idosos assistidos por um programa de Atenção Farmacêutica na Farmácia Popular do Brasil no município de Aracaju-SE. Revista de Ciências Farmacêuticas Básica e Aplicada, 33(2), 307-312.

Instituto Brasileiro De Geografia E Estatística - IBGE. Panorama de Lafaiete Coutinho - BA. https://cidades.ibge.gov.br/brasil/ba/lafaiete-coutinho/panorama. Instituto Brasileiro de Geografia e Estatística, IBGE. (2018). Projeção do Brasil por idade 2018 - $2030 . \quad$ Disponível em: https://ww2.ibge.gov.br/apps/populacao/projecao/

Kadam, U. T. (2011). Potential health impacts of multiple drug prescribing for older people: a case-control study. Br J Gen Pract., 61, 128-30.

Leão, I. N., Fernandes, B. D., Oliveira, B. G., Almeida, P. H. R. F., Lemos, G. S., Valasques Junior, G. L. \& Mascarenhas, C. H. M. (2020). Prevalência das interações medicamentosas potenciais em hipertensos atendidos na atenção primária. Rev. Aten. Saúde, 18(63), 05-13.

Loyola Filho, A. I. D., Uchoa, E. \& Lima-Costa, M. F. (2006). Estudo epidemiológico de base populacional sobre uso de medicamentos entre idosos na Região Metropolitana de Belo Horizonte, Minas Gerais, Brasil. Cadernos de Saúde Pública, 22, 2657-2667.

Lucchetti, G., Granero, A. L., Pires, S. L. \& Gorzoni, M. L. (2010). Fatores associados à polifarmácia em idosos institucionalizados. Revista Brasileira de Geriatria e Gerontologia, 13(1), 51-58.

Macedo, L. C., Rodrigues, C. \& Correa, L. M. (2016). Avaliação farmacoterápica em pacientes de uma instituição geriátrica da região centro-ocidental do Paraná, Brasil. SaBios-Revista de Saúde e Biologia, 11(1), 22-30.

Marin, M. J. S., Cecílio, L. C. O., Perez, A. E. W. U. F., Santella, F., Silva, C. B. A., Gonçalves Filho, J. R. \& Roceti, L. C. (2008). Caracterização do uso de medicamentos entre idosos de uma unidade do Programa Saúde da Família. Cad. Saúde Pública, 24(7), 1545-1555.

Marques, A. P. O, de Arruda, I. K. G., Leal, M. C. C. \& Espírito Santo, A. C. G. (2007). Envelhecimento, obesidade e consumo alimentar em idosos. Revista Brasileira de Geriatria e Gerontologia, 10(2), 231-242.

Mercedes, G. S., do Carmo Mercedes, B. P., de Oliveira, J. C. N., Sakamoto, L. M. \& Da Silva, N. M. R. R. (2013). Análise do perfil farmacoterapêutico e doenças prevalentes em pacientes idosos atendidos no hospital universitário de Ribeirão Preto-SP. Infarma-Ciências Farmacêuticas, 25(4), 88-192.

Morais, D. B., Paolinelli, J. P. V., Dâmaso, S. de F. T., Baldoni, A. O., \& Otoni, A. (2021). Influence of polypharmacy and use of inappropriate medication for the elderly on the glomerular filtration rate. Research, Society and Development, 10(4), e31810414239.

Neto, J. A. C., Delgado, A. A. A., Galvão, C. C. G. D., Machado, S. J. M., Bicalho, T. C. \& Oliveira, T. D. (2012). Uso de medicamentos por idosos de Juiz de Fora: um olhar sobre a polifarmácia. HU Revista, 37(3), 305-13.

Oliveira, M. A. D., Francisco, P. M. S. B., Costa, K. S \& Barros, M. B. D. A. (2012). Automedicação em idosos residentes em Campinas, São Paulo, Brasil: prevalência e fatores associados. Cad. Saúde Pública, 28, 335-345. 
Research, Society and Development, v. 10, n. 9, e29210918069, 2021

(CC BY 4.0) | ISSN 2525-3409 | DOI: http://dx.doi.org/10.33448/rsd-v10i9.18069

Ribeiro, L. C. C., Alves, P.B. \& De Meira, E. P. (2009). Percepção dos idosos sobre as alterações fisiológicas do envelhecimento. Ciência, Cuidado e Saúde, $8(2), 220-227$.

Rodrigues, D. S., Nery, S. B. M., Melo, G. A. de ., Mendes, J. S. A., Oliveira, G. A. L. de, \& Costa Neto, A. M. da . (2021). Impacts caused by polypharmacy on the elderly: an integrative review. Research, Society and Development, 10(2), e28810212263.

Sales, A. S., Sales, M. G. S. \& Casotti CA. (2017). Perfil farmacoterapêutico e fatores associados à polifarmácia entre idosos de Aiquara, Bahia, em 2014. Epidemiologia e Serviços de Saúde, 26(1), 121-132.

Sociedade Brasileira de Cardiologia, SBC. (2016). VII Diretriz brasileira de hipertensão. Arq Bras Cardiol., 107(3).

Veras, R. P. (2011). Estratégias para o enfrentamento das doenças crônicas: um modelo em que todos ganham. Revista Brasileira de Geriatria e Gerontologia, 14(4), 779-786.

World Health Organization, WHO (2019). Medication Without Harm - Global Patient Safety Challenge on Medication Safety. Medication Safety in Polypharmacy. Geneva: World Health Organization.

World Health Organization, WHO. (2000). Collaborating Centre for drug Statistics Metodhology. Anatomical Therapeutic Chemical (ATC) classification index with Defined Daily Doses (DDD’s). Oslo.

World Health Organization, WHO. (2002). Active Ageing - A Police Framework. A Contribution of the World Health Organization to the second United Nations World Assembly on Aging. Madrid, Spain.

World Health Organization, WHO. (2006). The safety of medicines in public health programmes: pharmacovigilance an essential tool. Geneva. 